\title{
Are Complete Blood Count Indices Different in Twins with the Diagnosis of Intrahepatic Cholestasis of Pregnancy?
}

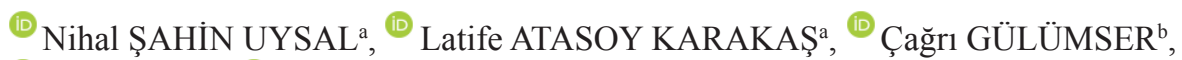 \\ (1) Sertaç ESINa ${ }^{1}$ Filiz F. YANIK ${ }^{\mathrm{a}}$ \\ aDepartment of Obstetrics and Gynecology, Başkent University Faculty of Medicine, Ankara, TURKEY \\ 'Private Clinic, Ankara, TURKEY
}

\begin{abstract}
Objective: To compare complete blood count (CBC) indices in twin pregnancies with and without the diagnosis of intrahepatic cholestasis of pregnancy (ICP). Material and Methods: This study was a retrospective case-control study of twin pregnancies complicated with ICP. The study and control groups included 36 twin pregnancies in each, respectively. The study and control groups were compared with each other with respect to the CBC indices (primary outcome measures of the study), maternal and newborn characteristics and pregnancy outcomes (secondary outcome measures). Statistical analyses were performed by using SPSS for Windows, version 22.0. Results: There were significant differences between the study and control groups regarding mean maternal age and median gestational age at delivery ( $\mathrm{p}<0.05$ ). Mean birthweight was significantly lower in the study group compared to control group ( $<<0.001)$. Neonatal intensive care unit (NICU) admission was significantly more frequent in the study group than in the control group ( $75 \%$ vs $33.3 \%$; $<0.001)$. The white blood cell (WBC), neutrophil and monocyte counts, neutrophil to lymphocyte ratios (NLRs) and monocyte to lymphocyte ratios (MLRs) were significantly lower, while mean platelet volume (MPV), aspartate aminotransferase (AST) and alanine aminotransferase (ALT) values were significantly higher in the study group than in the control group $(\mathrm{p}=0.002, \mathrm{p}=0.001, \mathrm{p}=0.004, \mathrm{p}=0.008, \mathrm{p}=0.006, \mathrm{p} \leq 0.001, \mathrm{p} \leq 0.001, \mathrm{p} \leq 0.001$, respectively). Conclusion: The NLRs and MLRs were significantly lower, while MPV was significantly higher in twin pregnancies with the diagnosis of ICP, than that of controls and ICP was found to be an independent predictor for increased MPV and decreased MLR values, irrespective of the other variables associated with MPV and MLR.
\end{abstract}

Keywords: Intrahepatic cholestasis of pregnancy; twin pregnancies

Intrahepatic cholestasis of pregnancy (ICP) is a pregnancy-specific liver disease that is presented by increased serum bile acids and liver transaminases and usually occurs in the second and third trimesters. ${ }^{1}$ ICP presents with pruritus beginning in the palmoplantar areas and spreads to the limbs and trunk. ${ }^{1}$ The incidence of ICP ranges from $0.2 \%$ to $>27 \%$ in different geographical regions and ethnic groups of the world. ${ }^{2}$ ICP incidence in twin pregnancies is two times than that of singleton pregnancies. ${ }^{3}$ Multiple gestation, itself, has been linked to the development of ICP. ${ }^{4}$ Not only fetal complications such as meconium-stained amniotic fluid, low birth weight, fetal distress and dysrhythmia, intrauterine death, preterm birth but also obstetric complications such as gestational diabetes, preeclampsia, preterm labor and postpartum hemorrhage increase in women with ICP. ${ }^{1,5-8}$

Although genetic, environmental, dietetic, hormonal and inflammatory factors are defined in disease pathogenesis, the etiology of ICP is not known precisely. ${ }^{2}$ It was reported that high bile acid level was involved in the ethiopathogenesis of cholestatic liver disease by triggering the inflammatory response, and proinflammatory cytokines were formed following the activation of inflammatory cells in the liver. ${ }^{5,9,10}$

The neutrophil-to-lymphocyte ratio (NLR), monocyte-to-lymphocyte ratio (MLR), platelet-to-

Correspondence: Nihal ŞAHIN UYSAL

Department of Obstetrics and Gynecology, Başkent University Faculty of Medicine, Ankara, TURKEY

E-mail: drnihalsahin@gmail.com

Peer review under responsibility of Journal of Clinical Obstetrics \& Gynecology. 
lymphocyte ratio (PLR) and mean platelet volume (MPV) are hematologic inflammatory markers. Some reports in the literature indicate that calculating the ratio of subtypes of blood cells, such as NLR, PLR and MPV could have prognostic significance for diseases associated with chronic lowgrade inflammation. ${ }^{11-14}$ Since these inflammatory markers are easily accessible and calculable parameters, those can be promising diagnostic tools in diseases related to chronic low-grade inflammation, such as ICP. ${ }^{11-14}$

NLR, PLR and MPV have been studied in singleton pregnancies with the diagnosis of ICP. ${ }^{5,14}$ Kırbaş et al., reported that NLR was found to be significantly higher in women with the diagnosis of ICP compared to that in women with normal pregnancies whereas Abide et al., reported that NLR was not significantly different between study and control groups, however MPV and PLR were significantly higher in the study group than in the control group. ${ }^{5,14}$

To the best of our knowledge, complete blood count $(\mathrm{CBC})$ indices reflecting inflammatory processes have not been studied as for an association with ICP in twin pregnancies. Therefore, we aimed to compare $\mathrm{CBC}$ indices in twin pregnancies with and without the diagnosis of ICP.

\section{MATERIAL AND METHODS}

This study was a retrospective case-control study of twin pregnancies complicated with ICP and those delivered at Başkent University Ankara Hospital between January 1, 2011 and December 31, 2019. The study group consisted of 36 twin pregnancies with the diagnosis of ICP and the control group included 36 twin pregnancies without the diagnosis of ICP. Inclusion criteria of the study were as follows: i) Pruritus without rash in various parts of the body, ii) Increased fasting total bile acid level (TBA) $(\geq 10$ $\mu \mathrm{mol} / \mathrm{L}$ ) and/or liver transaminase levels in the blood sample. Singleton pregnancies, TBA $<10 \mu \mathrm{mol} / \mathrm{L}$, missing $\mathrm{CBC}$ indices, patients with active hepatitis, fatty liver, gallstone disease, chronic inflammatory diseases, gestational or pregestational diabetes, preeclampsia, twin-specific complications, or fetal anomalies as well as maternal diseases including renal, malignancy, hematologic and autoimmune were excluded from the study. In addition, patients with any symptoms of systemic infections (cystitis, tonsillitis, flu, prolonged membrane rupture etc.) at the time of the blood collection were also excluded as infection affects the $\mathrm{CBC}$ indices.

Medical records were retrospectively reviewed for demographic variables and clinical data such as maternal age, gravidity, parity, gestational age at delivery, birth weight, and newborn outcome [admission to the neonatal intensive care unit (NICU) and maternal complete blood count (CBC) indices including white blood cell (WBC) count, hemoglobin $(\mathrm{Hb})$, hematocrit $(\mathrm{Hct})$, platelets (PLT), neutrophils, lymphocytes, NLR, MLR, PLR and MPV]. The study and control groups were compared with each other regarding the $\mathrm{CBC}$ indices (primary outcome measures of the study), maternal and newborn characteristics and pregnancy outcomes (secondary outcome measures). CBC indices, TBA levels and liver transaminases reported at the time of the diagnosis of ICP formed the laboratory data of the study group. Gestational week-matched reports of $\mathrm{CBC}$ indices and liver transaminases formed the laboratory data of control group.

$\mathrm{CBC}$ indices were determined using a CELLDYN Ruby hematology analyzer and TBA levels were determined by enzymatic assay.

Statistical analyses were performed by using SPSS for Windows, version 22.0. The Student's t-test, the Mann-Whitney $U$ test, chi-square test and Fisher's exact test were used where appropriate.

Correlation analyses of the hematologic inflammatory markers (NLR, MLR, PLR and MPV) with different clinical and laboratory variables were performed in the whole group. Where relevant, Pearson and Spearman tests were used. Multiple linear regression model was assessed to identify the independent predictors for elevated or decreased hematologic inflammatory marker values. A p value of $<0.05$ was considered statistically significant.

Institutional review board approval was obtained and the universal principles of the Helsinki Declaration were applied. 


\section{RESULTS}

The study and control groups included 36 twin pregnancies in each, respectively. We compared the demographic and baseline characteristics of the study and control groups (Table 1). There were significant differences between the study and control groups with respect to mean maternal age and median gestational age at delivery $(p<0.05)$. Mean birth weight was significantly lower in the study group than in the control group $(p<0.001)$. There were not any statistically significant differences between the study and control groups regarding median gravidity, parity and the chorionicity and conception types ( $p>0.05)$. NICU admission was significantly more frequent in the study group compared to control group ( $75 \%$ vs $33.3 \% ; \mathrm{p}<0.001)$. In the study group, median gestational age at diagnosis of ICP was 32 weeks (12-36).

Laboratory values are reported in Table 2 . The WBC, neutrophil and monocyte counts, NLRs and MLRs were significantly lower, while MPV, aspartate aminotransferase (AST), and alanine aminotransferase (ALT) values were significantly higher in the study group than in the control group $(\mathrm{p}=0.002$, $\mathrm{p}=0.001, \mathrm{p}=0.004, \mathrm{p}=0.008, \mathrm{p}=0.006, \mathrm{p} \leq 0.001$, $\mathrm{p} \leq 0.001, \mathrm{p} \leq 0.001$, respectively). Hb, Hct, PLT, lym- phocyte, eosinophil, basophil counts and PLRs were not significantly different between the study and control groups ( $p>0.05$, Table 2$)$. The mean TBA value was $26.16 \pm 16.21$ (range:10-71.80) in the study group (Table 2).

The bivariate analyses between hematologic inflammatory markers (NLR, MLR, PLR and MPV) and maternal demographic characteristics, clinical pregnancy outcomes and laboratory parameters with statistically significant differences between two groups, revealed significant correlation between NLR and AST, ALT ( $\mathrm{p}=0.009$ and $\mathrm{p}=0.039$, respectively); MLR and mean birth weight $(\mathrm{p}=0.039)$; MPV and gestational age at delivery, ALT and monocyte count ( $p=0.019, p=0.007$ and $p=0.026$, respectively) (Table $3)$. There were moderate, but significant, negative correlations between TBA and NLR, MLR and PLR $(\mathrm{r}=-0.48, \quad \mathrm{p}=0.003, \quad \mathrm{r}=-0.40, \quad \mathrm{p}=0.015, \quad \mathrm{r}=-0.41$, $\mathrm{p}=0.013$, respectively). The correlation analyses between hematologic inflammatory markers (NLR, MLR, PLR and MPV) and TBA are summarized in Table 4. ALT, AST and TBA were not included in the multivariate analysis that they were used in the laboratory criteria of the ICP diagnosis. Multivariate analysis, performed thereafter by including the parameters correlated with MLR and MPV-mean

TABLE 1: Maternal and newborn characteristics and pregnancy outcomes of the study and control groups.

\begin{tabular}{|c|c|c|c|}
\hline & $\begin{array}{c}\text { Study group } n=36 \\
\text { Median (range) } \\
\text { Mean } \pm S D \text { (range) }\end{array}$ & $\begin{array}{c}\text { Control group n=36 } \\
\text { Median (range) } \\
\text { Mean } \pm S D \text { (range) }\end{array}$ & $\mathrm{p}$ \\
\hline Maternal age (years) & $32.42 \pm 3.67(24-39)$ & $30.42 \pm 4.45(21-39)$ & $0.041^{\mathrm{a}}$ \\
\hline Gravidity & $1(1-3)$ & $1(1-6)$ & $0.909^{b}$ \\
\hline Parity & $0(0-2)$ & $0(0-3)$ & $0.107^{b}$ \\
\hline Gestational age at diagnosis of ICP & $32(12-36)$ & - & \\
\hline Gestational age at delivery (weeks) & $34(28-38)$ & $37(33-39)$ & $<0.001^{b}$ \\
\hline \multicolumn{4}{|l|}{ Chorionicity } \\
\hline Monochorionic & $2(5.5 \%)$ & $4(11.1 \%)$ & $0.674^{\circ}$ \\
\hline Dichorionic & $34(94.5 \%)$ & $32(88.8 \%)$ & \\
\hline \multicolumn{4}{|l|}{ Conception } \\
\hline Spontaneous & $12(33.33 \%)$ & $13(36.1 \%)$ & $0.804^{d}$ \\
\hline ICSI & $24(66.67 \%)$ & $23(63.8 \%)$ & \\
\hline \multirow[t]{2}{*}{ Mean birthweight (grams) } & $2175.56 \pm 423.62$ & $2683.89 \pm 279.52$ & \\
\hline & $(1198-3235)$ & $(2175-3275)$ & $<0.001^{\mathrm{a}}$ \\
\hline NICU admission & $54 / 72(75 \%)$ & $24 / 72(33.33 \%)$ & $<0.001^{d}$ \\
\hline
\end{tabular}

aStudent's t-test, ${ }^{\circ}$ Mann-Whitney U test, ${ }^{\circ}$ Fisher's exact test, ${ }^{\circ}$ Chi-square test

ICP: Intrahepatic cholestasis of pregnancy; ICSI: Intracytoplasmic sperm injection; NICU: Neonatal intensive care unit. 


\begin{tabular}{|c|c|c|c|}
\hline & Study group $n=36$ & Control group $n=36$ & \\
\hline & Mean $\pm S D$ (range) ${ }^{a}$ & Mean $\pm S D\left(\right.$ range) ${ }^{a}$ & \\
\hline Variables & Median (range) ${ }^{b}$ & Median (range) ${ }^{b}$ & $\mathrm{p}$ \\
\hline Hemoglobin (g/dL) & $12.41 \pm 1.30(8.90-15)$ & $12 \pm 1.14(8.80-14.40)$ & $0.168^{a}$ \\
\hline Hematocrit (\%) & $37.13 \pm 3.48(28.36-44.90)$ & $36.02 \pm 3.34(27.60-42.60)$ & $0.175^{a}$ \\
\hline Platelet $\left(x 10^{3} / \mathrm{L}\right)$ & $184.5(107-433)$ & $217(124-358)$ & $0.069^{b}$ \\
\hline WBC (cells/L) & $9.039 \pm 2.66(4.80-15.26)$ & $10.87 \pm 2.11(6.49-14.43)$ & $0.002^{\mathrm{a}}$ \\
\hline Neutrophil (cells/L) & $6.44 \pm 2.26(3.20-12.43)$ & $8.13 \pm 1.69(4.65-11)$ & $0.001^{\mathrm{a}}$ \\
\hline Lymphocyte (cells/L) & $1.86 \pm 0.57(0.83-3.67)$ & $1.87 \pm 0.52(0.68-2.91)$ & $0.965^{\mathrm{a}}$ \\
\hline Monocyte (cells/L) & $0.50 \pm 0.19(0.13-0.85)$ & $0.67 \pm 0.27(0.08-1.30)$ & $0.004^{\mathrm{a}}$ \\
\hline Eosinophil (cells/L) & $0.10(0-0.61)$ & $0.1(0-0.69)$ & $0.913^{b}$ \\
\hline Basophil (cells/L) & $0.04(0.02-0.12)$ & $0.05(0.01-0.15)$ & $0.493^{b}$ \\
\hline NLR & $3.36(1.53-8.10)$ & $4.48(2.38-15.63)$ & $0.008^{b}$ \\
\hline MLR & $0.28 \pm 0.11(0.12-0.62)$ & $0.37 \pm 0.16(0.06-0.75)$ & $0.006^{a}$ \\
\hline \multirow[t]{2}{*}{ PLR } & $112.86 \pm 37.27$ & $123.12 \pm 30.47$ & $0.205^{\mathrm{a}}$ \\
\hline & $(67.11-233.54)$ & $(55.0-184.54)$ & \\
\hline MPV (fL) & $10.07 \pm 2.02(7-14.80)$ & $8.50 \pm 1.75(5.96-13.40)$ & $0.001^{\mathrm{a}}$ \\
\hline Total bile acid ( $\mu \mathrm{mol} / \mathrm{L})$ & $26.16 \pm 16.21(10-71.80)$ & - & \\
\hline AST (U/L) & $39.5(15-213)$ & $19(6-30)$ & $<0.001^{b}$ \\
\hline ALT (U/L) & $58(9-327)$ & $15(6-34)$ & $<0.001^{b}$ \\
\hline
\end{tabular}

WBC: White blood cell count; NLR: Neutrophil-to-lymphocyte ratio; MLR: Monocyte-to-lymphocyte ratio; PLR: Platelet-to-lymphocyte ratio; MPV: Mean platelet volume; AST: Aspartate aminotransferase; ALT: Alanine aminotransferase.

\begin{tabular}{|c|c|c|c|c|c|c|}
\hline \multirow[b]{2}{*}{ Variables } & \multicolumn{2}{|c|}{ NLR } & \multicolumn{2}{|c|}{ MLR } & \multicolumn{2}{|c|}{ MPV } \\
\hline & $r$ & $p$ & $r$ & $\mathrm{p}$ & $r$ & $p$ \\
\hline Gestational age at delivery & & & & & -0.27 & 0.019 \\
\hline Mean birth weight & & & 0.24 & 0.039 & & \\
\hline Monocyte & & & & & -0.26 & 0.026 \\
\hline \multicolumn{7}{|l|}{ Total bile acid } \\
\hline AST & -0.35 & 0.009 & & & & \\
\hline ALT & -0.26 & 0.039 & & & 0.33 & 0.007 \\
\hline
\end{tabular}

NLR: Neutrophil-to-lymphocyte ratio; MLR: Monocyte-to-lymphocyte ratio; MPV: Mean platelet volume; AST: Aspartate aminotransferase; ALT: Alanine aminotransferase.

birth weight, gestational age at delivery and monocyte count- in the model, showed that ICP was an independent predictor for increased MPV and decreased MLR values, irrespective of the other variables associated with MPV and MLR ( $\mathrm{p}=0.003$ and $\mathrm{p}=0.020$, respectively).

\section{DISCUSSION}

To the best of our knowledge, hematologic inflammatory markers (NLR, MLR, PLR and MPV) have not been studied as for an association with ICP in twin pregnancies. Although the etiology of ICP is complicated and incompletely understood, inflam-
TABLE 4: Correlation analyses between hematologic inflammatory markers (NLR, MLR, PLR and MPV) and TBA in the study group.

\begin{tabular}{|lcc|}
\hline & \multicolumn{2}{c|}{ TBA } \\
Variables & $\mathbf{r}$ & $\mathbf{p}$ \\
NLR & -0.48 & 0.003 \\
MLR & -0.40 & 0.015 \\
PLR & -0.41 & 0.013 \\
MPV & 0.03 & 0.873 \\
\hline
\end{tabular}

NLR: Neutrophil-to-lymphocyte ratio; MLR: Monocyte-to-lymphocyte ratio; PLR: Platelet-to-lymphocyte ratio; MPV: Mean platelet volume; TBA: Total bile acid.

matory markers and immunological mechanisms come to the fore in recent studies. ${ }^{9,10,15}$ The initiator 
mechanism of the inflammatory response in the liver in patients with the diagnosis of ICP is not known. ${ }^{10}$ Gujral et al., reported that inflammatory cells such as neutrophils were documented to be activated and recruited into the liver during obstructive cholestasis, causing severe hepatic injury. ${ }^{16}$

Increased adverse fetal outcomes and generally not predicting these results make the management of this pregnancy specific disease difficult. In this study, we aimed to investigate the associations between the easily calculable hematologic inflammatory markers (NLR, MLR, PLR and MPV) with/out the diagnosis of ICP in twin pregnancies. These inflammatory markers can be calculated from CBC indices, those can be promosing diagnostic tools in diseases related to chronic low-grade inflammation, including ICP. ${ }^{11-14}$ The present study found that the NLRs and MLRs were significantly lower, while MPV was significantly higher in the study group than in the control group ( $\mathrm{p}=0.008, \mathrm{p}=0.006$ and $\mathrm{p}=0.001$, respectively). There were no statistically significant differences between two groups regarding PLRs $(p=0.205)$ (Table 2).

Abide et al., found that MPV values were significantly higher in patients with the diagnosis of ICP in singleton pregnancies than that of the controls, consistently with our results in twin pregnancies, but they found significantly higher PLR values in the study group than in the control group, contradictory to our results. ${ }^{14}$

There are conflicting results in the literature regarding WBC, neutrophil, lymphocyte counts and NLRs in singleton pregnancies with the diagnosis of ICP compared to controls. Abide et al., found that WBC counts were significantly higher while neutrophil and lymphocyte counts were significantly lower in the study group than in the control group, however Biberoğlu et al., Kırbaş et al. and Vural Yilmaz et al., found no significant differences with regard to WBC counts between two groups. ${ }^{5,14,17,18}$ Kırbaş et al., also found that neutrophil counts and NLRs were significantly higher while lymphocyte counts were significantly lower in the study group than in the control group, respectively. ${ }^{5}$ In our study WBC, neutrophil and monocyte counts were signifi- cantly lower in the study group than in the control group,while lymphocyte counts were not significantly different between two groups (Table 2).

Consistent with the literature in singleton pregnancies with the diagnosis of ICP, in our study there were no significant differences between two groups in twin pregnancies regarding the $\mathrm{Hb}$, Hct and PLT values. ${ }^{14,18}$

Klement et al., compared NLR, PLR and $\mathrm{Hb}$ values between low-risk and high-risk pregnancies (including multiple pregnancies), and investigated the reference values for NLR and PLR according to the trimesters. ${ }^{19}$ They reported that mean PLR and NLR values did not differ significantly between the groups by trimester or due to the high-risk pregnancy condition. Mean $\mathrm{Hb}$ values were reported to be $12.26 \mathrm{~g} / \mathrm{dL}$, $11.13 \mathrm{~g} / \mathrm{dL}$ and $11.44 \mathrm{~g} / \mathrm{dL}$ in the first, second and third trimesters, respectively. However, $\mathrm{Hb}$ values did not differ according to the presence of high-risk pregnancy condition. ${ }^{19}$

Batsry et al., reported that ICP was an additional factor leading to higher premature birth rates in twins $;{ }^{20}$ in accordance with the findings of Batsry et al., when comparing the study and control groups in respect of the study's secondary outcome measures, gestational age at delivery was significantly earlier and in parallel, mean birth weights of the neonates were significantly lower and the rate of the NICU admission were significantly higher in the study group than in the control group (Table 1). Consistently with the results described by Liu et al., mean maternal age was significantly higher in patients with the diagnosis of ICP than that of controls in our study. ${ }^{21}$

Correlation analyses revealed significant correlations between NLR and AST, ALT; MLR and mean birth weight; MPV and gestational age at delivery, ALT and monocyte count. There were moderate, but significant, negative correlations between TBA and NLR, MLR and PLR. Once we conducted the multivariate analysis, ICP was found to be an independent predictor for increased MPV and decreased MLR values, irrespective of the other variables associated with MPV and MLR.

Carefully data collection from medical records, detailed pregnancy follow-up and being a single- 
center study using the same diagnostic criteria and management protocol of ICP for all patients are the strengths of this study. The study's primary limitation is its retrospective design, which limits the ability to monitor for potential confounders and also the sample size of the study was small, partly due to the study population (twin pregnancies with the diagnosis of ICP). Although some of the current study results supported our initial hypothesis (inflammatory process in ICP etiopathogenesis), some did not. To our knowledge, as this is the first study evaluating hematologic inflammatory markers (NLR, MLR, PLR and MPV) in twin pregnancies with the diagnosis of ICP, our study results remain to be supported with further studies. Also, the reference values for $\mathrm{CBC}$ indices in twin pregnancies may be studied in further studies.

\section{CONCLUSION}

The NLRs and MLRs were significantly lower, while MPV was significantly higher in twin pregnancies with the diagnosis of ICP, than that of controls and ICP was found to be an independent predictor for increased MPV and decreased MLR values, irrespective of the other variables associated with MPV and MLR.

\section{Informing}

Due to the presence of the name of the journal editor's among the authors, the assessment process of the study was conducted by the guest editor.

\section{Source of Finance}

During this study, no financial or spiritual support was received neither from any pharmaceutical company that has a direct connection with the research subject, nor from a company that provides or produces medical instruments and materials which may negatively affect the evaluation process of this study.

\section{Conflict of Interest}

No conflicts of interest between the authors and / or family members of the scientific and medical committee members or members of the potential conflicts of interest, counseling, expertise, working conditions, share holding and similar situations in any firm.

\section{Authorship Contributions}

Idea/Concept: Nihal Şahin Uysal; Design: Nihal Şahin Uysal, Latife Atasoy Karakaş; Data Collection and/or Processing: Nihal Şahin Uysal, Latife Atasoy Karakaş; Analysis and/or Interpretation: Çağrl Gülümser, Nihal Şahin Uysal; Literature Review: Çă̆rl Gülümser; Writing the Article: Nihal Şahin Uysal; Critical Review: Filiz F. Yanık; References and Fundings: Sertaç Esin, Filiz F. Yanık; Materials: Çağrı Gülümser, Sertaç Esin, Filiz F. Yanı.

\section{REFERENCES}

1. Geenes V, Williamson C. Intrahepatic cholestasis of pregnancy. World J Gastroenterol. 2009;7;15(17):2049-66. [Crossref] [PubMed] [PMC]

2. Williamson C, Geenes V. Intrahepatic cholestasis of pregnancy. Obstet Gynecol. 2014;124(1):120-33. [Crossref] [PubMed]

3. Liu S, Li L, Liu H. Clinical analysis of 57 cases of intrahepatic cholestasis of pregnancy with twins. Sichuan Med. 2010;12(31):1814-5.

4. Lausman AY, Al-Yaseen E, Sam D, Nitsch R, Barrett JFR, Chan WS, et al. Intrahepatic cholestasis of pregnancy in women with a multiple pregnancy: an analysis of risks and pregnancy outcomes. J Obstet Gynaecol Can. 2008;30(11):1008-13. [Crossref] [PubMed]

5. Kirbas A, Biberoglu E, Daglar K, Ýskender C, Erkaya S, Dede H, et al. Neutrophil-to-lymphocyte ratio as a diagnostic marker of intrahepatic cholestasis of pregnancy. Eur J Obstet
Gynecol Reprod Biol. 2014;180:12-5. [Crossref] [PubMed]

6. Rook M, Vargas J, Caughey A, Bacchetti P, Rosenthal $P$, Bull $L$, et al. Fetal outcomes in pregnancies complicated by intrahepatic cholestasis of pregnancy in a Northern California cohort. PLoS One. 2012;7(3):e28343. [Crossref] [PubMed] [PMC]

7. Gul A, Gungorduk K, Turan I, Yildirim G, Gedikbasi A, Özdemir A, et al. Prenatal diagnosis of 22q11.2 deletion syndrome in twin pregnancy: a case report. J Clin Ultrasound. 2013;41(S1):6-9. [Crossref] [PubMed]

8. Shemer EW, Marschall HU, Ludvigsson JF, Stephansson O. Intrahepatic cholestasis of pregnancy and associated adverse pregnancy and fetal outcomes: a 12-year populationbased cohort study. BJOG. 2013;120(6):71723. [Crossref] [PubMed]

9. Kosters A, Karpen SJ. The role of inflamma- tion in cholestasis: clinical and basic aspects. Semin Liver Dis. 2010;30(2):186-94. [Crossref] [PubMed] [PMC]

10. Allen $\mathrm{K}$, Jaeschke H, Copple BL. Bile acids induce inflammatory genes in hepatocytes: a novel mechanism of inflammation during obstructive cholestasis. Am J Pathol. 2011;178(1):175-86. [Crossref] [PubMed] [PMC]

11. Tamhane UU, Aneja S, Montgomery D, Rogers EK, Eagle KA, Gurm HS, et al. Association between admission neutrophil to lymphocyte ratio and outcomes in patients with acute coronary syndrome. Am J Cardiol. 2008;15;102(6):653-7. [Crossref] [PubMed]

12. Azab B, Jaglall N, Atallah PJ, Lamet A, Raja-Surya V, Farah B, et al. Neutrophillymphocyte ratio as a predictor of adverse outcomes of acute pancreatitis. Pancreatology. 2011;11(4):445-52. [Crossref] [PubMed] 
13. Torun $\mathrm{S}$, Tunc BD, Suvak B, Yildiz H, Tas A, Sayilir $A$, et al. Assessment of neutrophil-lymphocyte ratio in ulcerative colitis: a promising marker in predicting disease severity. Clin Res Hepatol Gastroenterol. 2012;36(5):491-7. [Crossref] [PubMed]

14. Abide ÇY, Vural F, Kılıççı Ç, Bostancı Ergen E, Yenidede I, Eser A, et al. Can we predict severity of intrahepatic cholestasis of pregnancy using inflammatory markers? Turk J Obstet Gynecol. 2017;14(3):160-5. [Crossref] [PubMed] [PMC]

15. Miossec $P$, Kolls JK. Targeting IL-17 and TH17 cells in chronic inflammation. Nat Rev Drug Discov. 2012;11(10):763-76. [Crossref] [PubMed]
16. Gujral JS, Farhood A, Bajt ML, Jaeschke H. Neutrophils aggravate acute liver injury during obstructive cholestasis in bile duct-ligated mice. Hepatology. 2003;38(2):355-63. [Crossref] [PubMed]

17. Biberoglu E, Kirbas A, Daglar K, Kara O, Karabulut E, Yakut HÝ, et al. Role of inflammation in intrahepatic cholestasis of pregnancy. J Obstet Gynaecol Res. 2016;42(3):252-7. [Crossref] [PubMed]

18. Yilmaz ZV, Turkmen GG, Daglar K, Yýlmaz E, Kara O, Uygur D, et al. Elevated red blood cell distribution width is associated with intrahepatic cholestasis of pregnancy. Ginekol Pol. 2017;88(2):75-80. [Crossref] [PubMed]

19. Klement AH, Hadi E, Asali A, Shavit T, Wiser A,
Haikin E, et al. Neutrophils to lymphocytes ratio and platelets to lymphocytes ratio in pregnancy: a population study. PLoS One. 2018;22;13(5): e0196706. [Crossref] [PubMed] [PMC]

20. Batsry L, Zloto K, Kalter A, Baum M, MazakiTovi S, Yinon Y. Perinatal outcomes of intrahepatic cholestasis of pregnancy in twin versus singleton pregnancies: is plurality associated with adverse outcomes? Arch Gynecol Obstet. 2019;300(4):881-7. [Crossref] [PubMed]

21. Liu X, Landon MB, Chen Y, Cheng W. Perinatal outcomes with intrahepatic cholestasis of pregnancy in twin pregnancies. J Matern Fetal Neonatal Med. 2016;29(13):2176-81. [Crossref] [PubMed] 